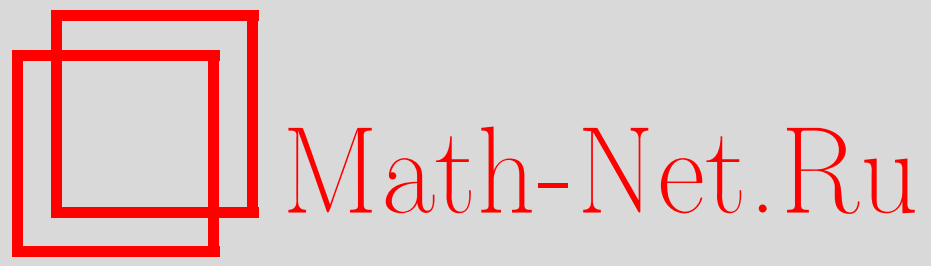

В. И. Арнольд, Слабые асимптотики чисел решений диофантовых задач, Функи. анализ и его прил., 1999, том 33, выпуск 4, 65-66

DOI: https://doi.org/10.4213/faa381

Использование Общероссийского математического портала MathNet.Ru подразумевает, что вы прочитали и согласны с пользовательским соглашением

http://www. mathnet.ru/rus/agreement

Параметры загрузки:

IP : 35.173 .219 .12

26 апреля 2023 г., 15:16:11

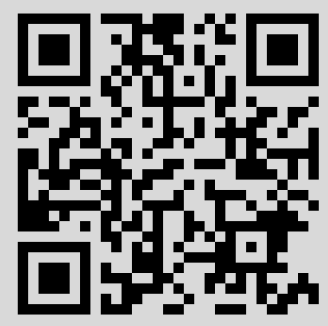




\title{
Слабые асимптотики чисел решений диофантовых задач*
}

\author{
(C) 1999. В. И. Арнольд
}

1. Введение. Простейшие примеры диофантовых задач, о которых пойдет речь, - определение числа точек мелкой решетки в области и проблема Сильвестра-Фробениуса о наименьшем числе $N\left(a_{1}, \ldots, a_{n}\right)$, начиная с которого все целые числа представляются в виде линейных комбинаций $k_{1} a_{1}+\cdots+k_{n} a_{n}$ заданных натуральных чисел $a_{1}, \ldots, a_{n}$ с целыми неотрицательными коэффициентами $k_{i}$.

Вторая задача решена Сильвестром для $n=2$ (ответ $N=\left(a_{1}-1\right)\left(a_{2}-1\right)$, $a_{1}$ и $a_{2}$ взаимно просты). Под слабой асимптотикой я хотел бы здесь понимать $N \sim a_{1} a_{2}$ (пренебрегая меньшими поправками).

В первой задаче асимптотику доставляет объем области, поделенный на объем основного параллелепипеда решетки. Но эта асимптотика лишь слабая потому, что область может иметь, скажем, плоскую грань с большим числом целых точек, что делает число точек в области неустойчивым, тогда как объемы устойчивы.

Идея состоит в том, чтобы формализовать эту ситуацию, сделав задачи с подобными неустойчивостями доступными средствам анализа, несмотря на теоретико-числовые флуктуации в исключительных случаях.

2. Слабые асимптотики. Под задачей я буду понимать вычисление функции $f$ от решетки $\Gamma$ в $\mathbb{R}^{n}$. Примеры: 1) $f=$ число точек решетки $Г$ в области, 2) $f=\sum U(\gamma)$ по $\gamma \in \Gamma$ для некоторого потенииала $U$.

Под асимптотикой я буду понимать исследование поведения $f$ при уплотнении решетки, например, поведения величины $f(\Gamma / M), M \rightarrow \infty$. Acuмnтотические формульи могут иметь, например, вид

$$
\lim _{M \rightarrow \infty} a(\Gamma / M)=c, \quad \lim _{M \rightarrow \infty} \frac{a(\Gamma / M)}{b(\Gamma / M)}=c
$$

(скажем, для $b=C(\Gamma) M^{d}$ и т.п.). Пределы здесь слабые: обычную сходимость доставляют только интегралы

$$
\lim _{M \rightarrow \infty} \int a(\Gamma / M) F(\Gamma) d \Gamma=c(F),
$$

где $F$ - гладкое размазывание решетки по окрестности точки Г. Конечно, предполагается сходимость $c(F)$ к $c$ для типичных $\delta$-образных размазываний $F$.

Легко доказывается

Теорема 1. Число Г-цельх точек в ограниченном многограннике слабо асимптотически пропорчионально объему многогранника.

*Частично поддержано РФФИ, грант 99-01-01109, и фондом Institute Universitaire de France. 
3. Перспективы. Здесь я обсуждаю предположительные применения этих идей к проблеме Фробениуса.

Зафиксируем отображение $a: \mathbb{R}^{n} \rightarrow \mathbb{R}, a\left(k_{1}, \ldots, k_{n}\right)=a_{1} k_{1}+\cdots+a_{n} k_{n}$, где $a_{i}$ - натуральные числа. Рассмотрим прямой образ меры на области $\{k \geqslant 0\}$

$$
F_{a}(x)=\text { число целых векторов }\left(k_{i}\right), k_{i} \geqslant 0, \text { для которых } a(k) \leqslant x .
$$

Аргументы, доказывающие теорему 1 , доставляют следующее утверждение:

Теорема 2. Число $F_{a}$ слабо асимптотически (при а $\rightarrow \infty$ ) равно объему соответствуюшего симплекса, $F_{a}(x) \sim(n !)^{-1} \prod\left(x / a_{i}\right)$.

Конечно, предполагается, что $\left\{a_{i}\right\}$ не имеют общего множителя.

Утоньшение решетки соответствует замене $a_{i}$ на $M A_{i}, x$ на $M X$, где $M \rightarrow \infty$ :

$$
F_{A}(X) \sim(n !)^{-1} \prod\left(X / A_{i}\right),
$$

причем слабость означает размазывание постоянных $a_{i}$.

ЗАмечАниЕ. Уже следствие $F_{A} \sim c M^{n}$ интересно.

Для проблемы Фробениуса нужно исследовать «производную» $\Delta_{a}(x)=$ $F_{a}(x+1)-F_{a}(x)$ : число $N(a)$ определено условием $\Delta_{a}(x) \geqslant 1$ при $x \geqslant N$.

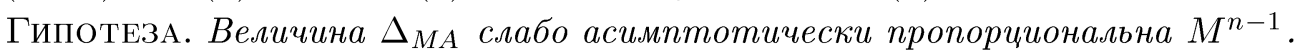

Трудность (оценки $\Delta$ снизу) по сравнению с теоремами лежит в возможности сушествования лакун среди точек $a(k)$ на оси $x$ вблизи $N$. Пренебрегая этими трудностями, получаем

$$
N \sim\left((n-1) ! \prod a_{i}\right)^{1 /(n-1)}, \quad N(M A) \sim M^{1 /(n-1)} N(A) .
$$

В случае Сильвестра $(n=2) N \sim a_{1} a_{2}$ действительно достаточно. В следующем случае $(n=3) N \sim \sqrt{2 a_{1} a_{2} a_{3}}$ кажется достаточным экспериментально.

Можно предвидеть универсальность статистики лакун $\left\{x: F_{a}(x)=0\right\}$. Для $n=2$ они антисимметричны: $x-$ лакуна, если и только если $N-1-x-$ не лакуна.

Математический институт им. В. А. Стеклова и CEREMADE, Universitè Paris-Dauphine

Поступило в редакцию 15 сентября 1999 г.

\title{
УДК 513.8
}

\section{Об условиях на производящий оператор равномерно ограниченной $C_{0}$-полугруппы операторов}

\author{
(c) 1999. А. М. Гомилко
}

Пусть $B$ - банахово пространство с нормой $\|\cdot\|$, а $B^{*}-$ его сопряженное с нормой $\|\cdot\|_{*}$. Через $(x, y)$ обозначим действие линейного непрерывного функционала $y \in B^{*}$ на вектор $x \in B$; в частности, если $B$ - гильбертово пространство, то $(x, y)$ - скалярное произведение в $B$. Все рассматриваемые далее операторы предполагаются линейными и действующими в $B$, а $I$ - тождественный оператор. Для оператора $A$ через $\sigma(A)$ обозначим его спектр, и пусть $R(A, \lambda)=(A-\lambda I)^{-1}, \lambda \notin \sigma(A)$, - резольвента оператора $A$. 